\title{
Applied iterative closest point algorithm to automated inspection of gear box tooth
}

\author{
Salim Boukebbab ${ }^{\mathrm{a}, *}$, Hichem Bouchenitfa ${ }^{\text {a }}$, Hamlaoui Boughouas ${ }^{\mathrm{a}}$, \\ Jean Marc Linares ${ }^{b, 1}$

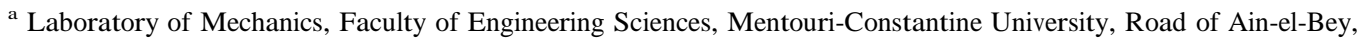 \\ 25000 Constantine, Algeria \\ ${ }^{\text {b }}$ EA(MS), Université de la Méditerranée, Institut Universitaire de Technologie, Avenue Gaston Berger, \\ 13625 Aix-en-Provence cedex 1, France
}

\begin{abstract}
The development of a complete system of inspection and quality control of manufactured parts requires the coordination of a set of complex processes allowing data acquisition, their dimensional evaluation and their comparison with a reference model. By definition, the parts inspection is the comparison between measurement results and the theoretical surfaces definition in order to check the conformity after manufacturing phase. The automation of this function is currently based on alignment methods of measured points resulting from an acquisition process and these nominal surfaces, in a way that they "fit best". The distances between nominal surface and measured points (i.e. form defects) calculated after alignment stages are necessary for the correction of the manufacturing parameters (Henke, Summerhays, Baldwin, Cassou, $\&$ Brown, 1999). In this work, a method for automated control based on association of complex surfaces to a cloud points using the Iterative Closest Point (I.C.P.) algorithm for alignment stage is proposed. An industrial application concerning a tooth gear manufactured in our country's tractor engines is presented.
\end{abstract}

Ó 2007 Elsevier Ltd. All rights reserved.

Keywords: CMMs; Complex surfaces; ICP; Gear; Manufacturing process

\section{Introduction}

The design and manufacture of complex surfaces became a current practice in industry. These surfaces can be conceived by a direct method based on the use of Computer Aided Design (CAD) software, or an indirect method which consists in a treatment of a discrete representation of an object model to obtain its CAD model. This last can be obtained throughout an acquisition process, allowing then a much more rapid safeguard,

\footnotetext{
* Corresponding author. Tel.: +213 318188 53; fax: +213 31818863.

E-mail address: boukebbab@yahoo.fr (S. Boukebbab).

${ }^{1}$ Tel.: +33442939096.
} 


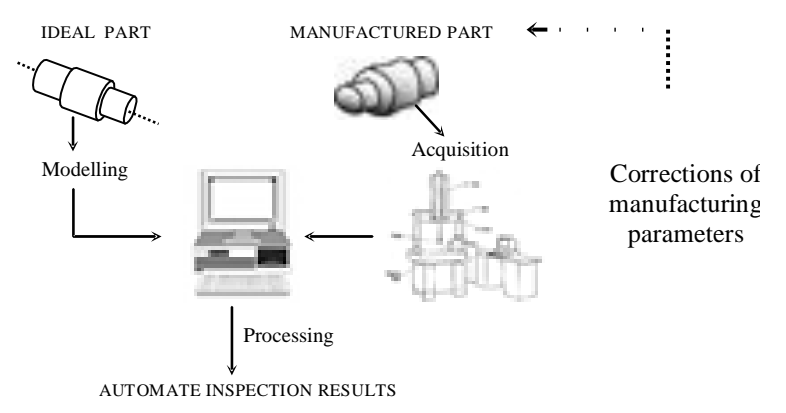

Fig. 1. Automation inspection case.

modification, manufacture, automatic inspection, prototypes checking and a much easier analysis (Lai \& Ueng, 2000).

The last years, the development process has covered all automated production phases, from design to the parts inspection passing by manufacture. Since the design and the manufacture of complex surfaces became a current practice in industry, then the problems related to the parts conformity are being felt more and more.

The automation and the flexibility of a three-dimensional measurement machine with or without contact have made a considerable reduction in the acquisition time and the measurements treatment. In the current state of the metrology software, the inspection of elementary surfaces (plane, cylinder, cone, etc.) became a very easy practice. On the other hand the inspection of complex surfaces remains a problem to overcome (Tucker \& Kurfess, 2003).

The ascending complexity of parts geometry and the need for reducing production costs impose the use of more powerful tools for the inspection of complex parts surfaces, for a better service functionality description during its assembly with the conjugate mechanism parts (Tholath \& Radhakrishnan, 1999). Our work is placed accordingly and consists to establish a procedure for modelling and inspecting complex parts surfaces, enabling the correction of relative deviations within production means (Fig. 1).

The method used is based on the iterative-closest-point (ICP) algorithm, which is a well-known method for registering a 3D set of points to a 3D model that minimizes the sum of squared residual errors between the set and the model. This choice is motivated by the robustness of this method and it is important to underline here that; no attempt to implement it within Coordinate Measuring Machines (CMMs) software has been reported in the three-dimensional metrology literature.

A numerical application treating the case of a tooth of the toothed wheel which equips the gear box tractor manufactured at the engines and tractors factory in our country is presented. The comparison between the real surface obtained by acquisition and the ideal model has led to the calculation of the form defects on the two flanks of the tooth gear.

\section{Problems and adopted algorithm}

The principle of the software of Coordinate Measuring Machines consists generally in individually associating an elementary mathematical model (plane, cylinder, etc) to each digitized surface. The function to be minimised is based on the distance $d_{i}$ between the digitized point $\mathbf{M}_{i}$ and the theoretical surface (Fig. 2).

As already pointed out in the introduction, in current state of the metrology software, the inspection of elementary surfaces (plane, cylinder, cone, etc.) is not a problem, and most CMMs correct remaining alignment deviations numerically (alignment means to evaluate an optimum transformation $\mathrm{T}$ mapping the measured points to the corresponding nominal points in a way that they "fit best") (Goch et al., 2003). On the other hand the inspection of surfaces which have geometries of a higher complexity like gears, sculptured surfaces etc. represents a major challenge (Goch \& Tschudi, 1992; Pommer, 2002). It is to this objective that our work is directed, and consists in the development of a procedure for modeling and inspecting complex surfaces with an aim of correcting the errors cumulated during the manufacturing phase (Portman \& Shuster (1997)). For this case, the ICP (Iterative Closest Point) algorithm method will be used. 


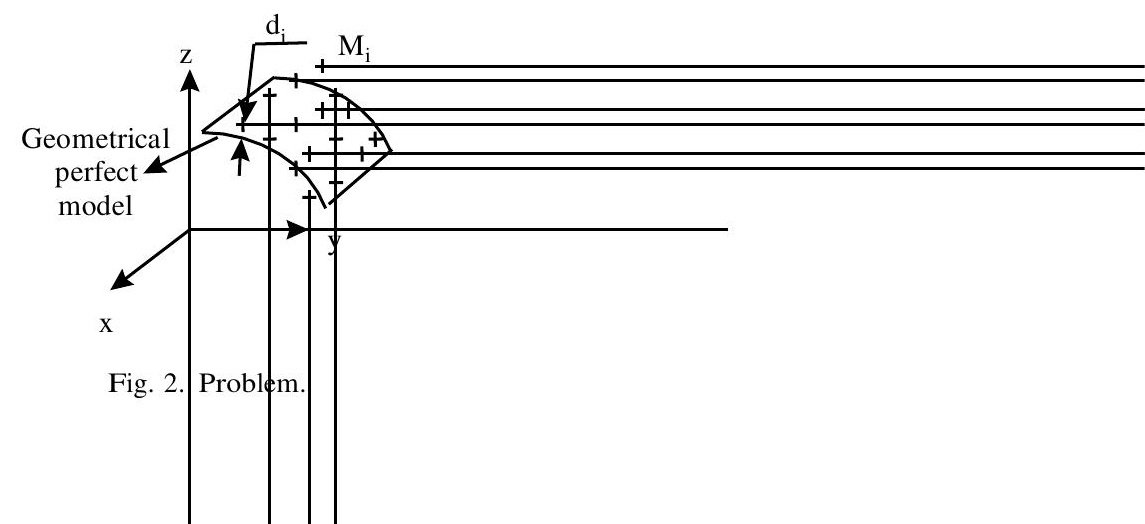

The iterative-closest-point (ICP) algorithm of Besl and Mc Kay (1992) is a well-known method for registering a 3D set of points to a 3D model that minimizes the sun of qquared residual errors between the set and the model, i.e. it finds a registration that is locally best in a least-squares sense (Bergevin, Laurendeau, \& Poussart, 1995; Ma \& Ellis, 2003). Its main goal is to find the optinhal r rgid transformation which will corresponds as well as possible a cloud points $\mathbf{P}$ to a geometrical model $\mathrm{M}$, using the singular value decomposition function (SVD) (Fig. 3).

The parameters of the rigid transformation between thø sфts of points $\mathrm{P}^{\mathrm{I}}$ and $\mathrm{P}^{\mathrm{II}}$ must minimize the cost function:

$$
\begin{aligned}
& 1 \\
& \mathrm{~N}_{\mathrm{s}} \\
& \mathrm{i}^{1} / 41
\end{aligned} \mathrm{kP}_{\mathrm{i}}^{00} \ddot{\mathbf{y}} \mathrm{T}_{\mathrm{t}} 1 \partial \mathrm{P}^{0} \mathrm{pk}^{2}
$$

Where: $\mathrm{P}_{\mathrm{i}}^{0}$ is a point from $\mathrm{P}^{\mathrm{I}} \mathrm{P}_{\mathrm{i}}^{00}$ is a point from $\mathrm{P}^{\mathrm{II}}$ associat $\mathrm{d}$ with $\mathrm{P}_{\mathrm{i}}^{0} \mathrm{~T}_{\mathrm{t}}$ the regid transformation.

A rigid transformation $T_{t}$ consists of the rotation rhatrix $[R]$ and the translation vector $\{\mathrm{T}\}$ giving the iterative transformation $\mathrm{P}_{\mathrm{i}}^{00}{ }_{1}^{1 / 4} / \mathrm{R} \mathrm{R}^{1} 1 \mathrm{P}^{0}{ }_{\mathrm{i}} \mathrm{p}$ fTg $\left(\mathrm{P}_{\mathrm{i}}^{0}\right.$ will be transforned into a point $\left.\mathrm{P}_{\mathrm{i}}^{00}\right)$.

This algorithm requires an initial estimate of the registration; because the computation speed and registration accuracy depend on how this initial estimate is chosen ( $\mathrm{Ma} \&$ Ellis, 2003). For this, we were mainly based on the algorithm proposed by Moron (1996) to which some changes have been made in order to make it more simple while keeping a maximum of its performances Fig. 4.

In this algorithm, we have to determine the six degrees of frłedom including the three for rotation and the other three for translation by ICP. While the three dimensional translation vector has simply three parameters as $\{\mathrm{T}\}=(\mathrm{tx}, \mathrm{ty}, \mathrm{tz})^{\mathrm{T}}$, the rotation matrix is apparently composed of nine elements which should go along with six conditions for orthonormality.

A simple iterative optimization based on the least square pinciple can not guarantee this orthonormality (Kaneko, Kondo, \& Miyamoto, 2003). Hence, ICP employs upit quaternion $\left(\mathrm{q}_{0} ; \mathrm{q}_{1} ; \mathrm{q}_{2} ; \mathrm{q}_{3}\right)$ for representing the rotation parameters in order to reduce this problem.

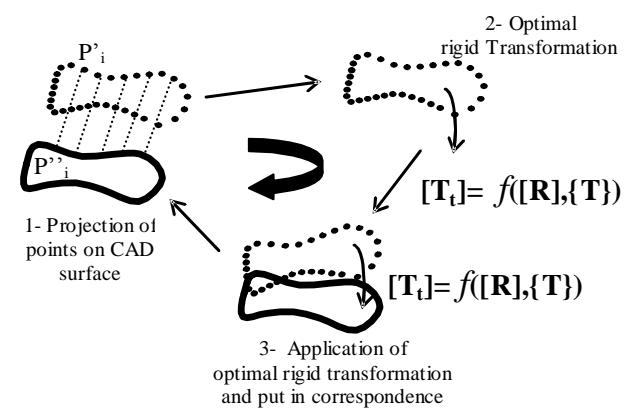

Fig. 3. Principal steps of the I.C.P. algorithm. 


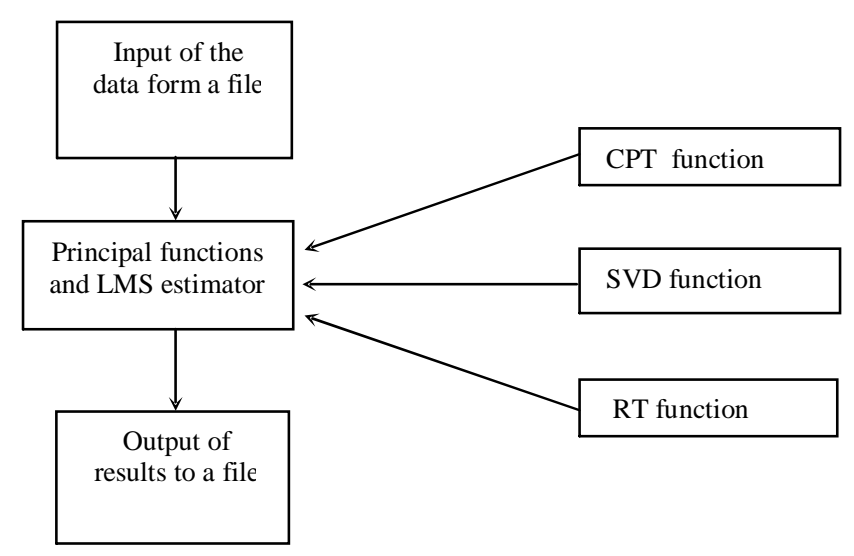

Fig. 4. Program structure.

The unit quaternion is used to compute a rotation about the unit vector $\mathrm{n}$ by an angle $\mathrm{h}$ :

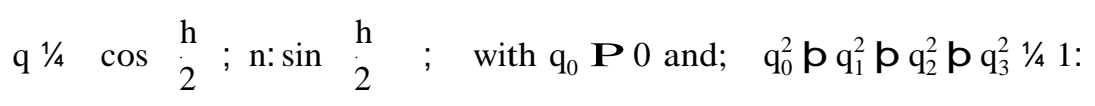

Then the rotation matrix $[R]$ is defined by:

$$
\begin{aligned}
& 2 q_{0}^{2} p q_{1}^{2} \ddot{\mathbf{y}} q_{2}^{2} \ddot{\mathbf{y}} q_{3}^{2} \quad 2 \partial q_{1} q_{2} \ddot{y} q_{0} q_{3} p \quad 2 \partial q_{1} q_{3} p q_{0} q_{2} p \quad 3
\end{aligned}
$$

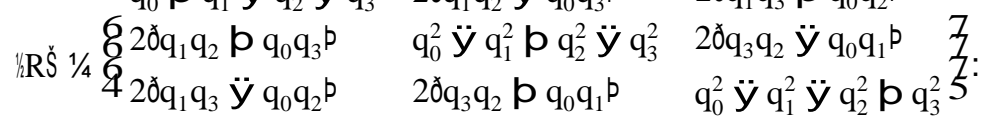

The optimal motion ([R]; $\{\mathrm{T}\})$ is computed by the unit quaternion method due to Horn (Eggert, Lorusso, \& Fisher, 1997). The same method was used in the original version of ICP (Besl \& McKay, 1992). There are different analytical ways to calculate the $3 \mathrm{D}$ rigid motion that minimises the sum of the squared distances between the corresponding points. In Eggert et al. (1997), four such techniques were compared and the unit quaternion method was found to be robust with respect to noise, stable in presence of degenerate data and relatively fast (Chetverikov, Stepanov, \& Krsek, 2005).

\section{Presentation of the algorithm}

Since the presentation of the I.C.P. algorithm by Besl and Mckay, many variants have been introduced, which aff ect one or more stages of the original algorithm to try to increase its performances specially accuracy and speed, giving birth to several alternatives of I.C.P. algorithm (Kaneko et al., 2003). Some of these variants (such as Rusinkiewicz et al. (2001)) expand also the abbreviation to the iterative corresponding point claiming that this would better suit the algorithm (Sablatnig \& Kampel, 2002). In order, to make a choice of an algorithm, several criteria should be checked: speed, accuracy, stability, robustness, and simplicity. The importance of the one or other of those criteria depends on the use and the application of the final program.

The development of a complete system of inspection and quality control of manufactured parts requires the coordination of a set of complex processes allowing data acquisition, their dimensional evaluation and their comparison with a reference model. For that it is essential to make profitable some conceptual knowledge relating not only to the object to be analyzed, but also to its environment. In our case, the objective of the present work consist in establishing an automation procedure for modelling and inspecting complex parts surfaces, enabling the correction of relative deviations within manufacturing parameters, then the criteria adopted are: speedy convergence, system robustness, and interface simplicity.

The new algorithm can be summarized by the following procedure: 
1. Make a random selection of a subset of points.

2. Calculate the projection of the selected points.

3. Calculate the optimal rigid transformation with SVD method.

4. Apply the transformation to the selected points.

5. Evaluate the quality of alignment by LMS estimator.

6. If alignment quality is good, calculate transformation and apply it to the whole of available points.

7. Repeat the steps from 1 to 6 until convergence.

The conceptual structure of our program is presented in Fig. 4.

We note here that the algorithm structure is very simple; it is made up of a principal program which contains a loop to carry out the iterations and an other one to estimate the quality of the rigid transformation by the LMS estimator (Least Median Squares) (Rousseuw \& Leroy, 1987). In this program we also find three call functions which are: the CPT function which calculates the projection of the points on the ideal model of surface in STL format (Fig. 5), the SVD function which calculates the optimal rigid transformation; and finally the RT function useful for calculating the initial rigid transformation; because as already pointed out, the algorithm requires an initial estimate solution of registration; and the computation speed and registration accuracy depend on how this initial estimate is chosen (Ma \& Ellis, 2003).

The STL format is generally obtained by a triangulation of an exact model using CAD software which gives a data file in STL format (Fig. 6). Where a Triangular facet is defined by the co-ordinates of the three vertexes and its normal directed towards the object free side.

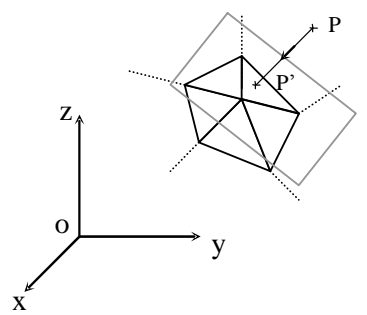

Fig. 5. Point projection on STL surface format.

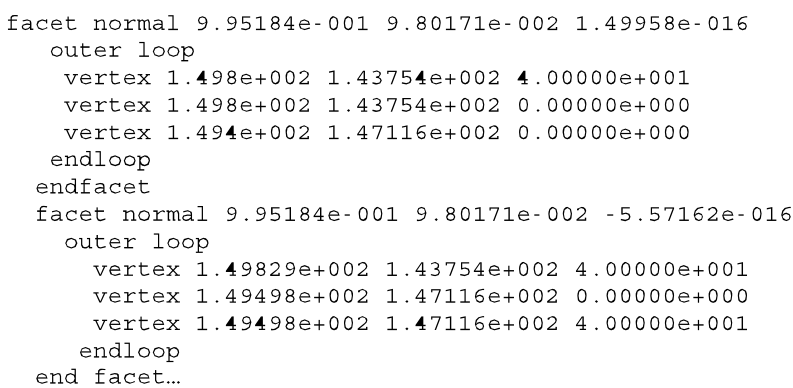

Fig. 6. Extract from an STL file.

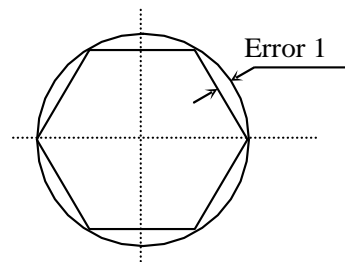

Approximation -1-

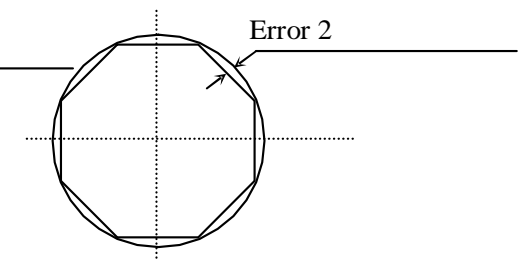

Approximation -2-

Fig. 7. Approximation errors according to triangle number. 
It should be noted that, the bigger is the number of triangles in STL model the less is the approximation errors (Fig. 7).

The number of triangles and their distributions are function of the surface curvature and the modelling tolerated error.

\section{Realization and performances test}

A data-processing model is realised to test the algorithm performances on a PC equipped with a microprocessor Pentium III, $1.33 \mathrm{Ghz}$ with 128 Mo of RAM. The adopted principle is easy; in the first stage one or more surfaces in STL format are generated in order to simulate the ideal model, in the second stage we apply a displacement to the co-ordinates of nodes to simulate measurement errors (Fig. 8a). By the application of a rigid transformation, the final transformation provided by the program realises alignment between the cloud points and the surface in STL format (Fig. 8c).

The program reliability is tested by considering two principal factors: the convergence and the necessary time for the program to be executed (Figs. 9 and 10).

In Fig. 9 the program converges quickly on a stable solution after a maximum of 05 iterations; after 10 iterations the alignment is practically perfect. Fig. 10 presents the curve characterizing the variation of the execution time according to the number of triangles, the execution time for 800 triangles for example is approximately $20 \mathrm{~s}$, a value which is rather weak and can be reduced if a better strategy for the calculation of projections by the CPT function is adopted.

\section{Numerical application on a gear tooth}

Today's gear metrology has to meet similar challenges as other geometric measurements, performed e.g. by CMMs or form testers: the alignment, the tactile or non-contact probing of selected surfaces areas, the
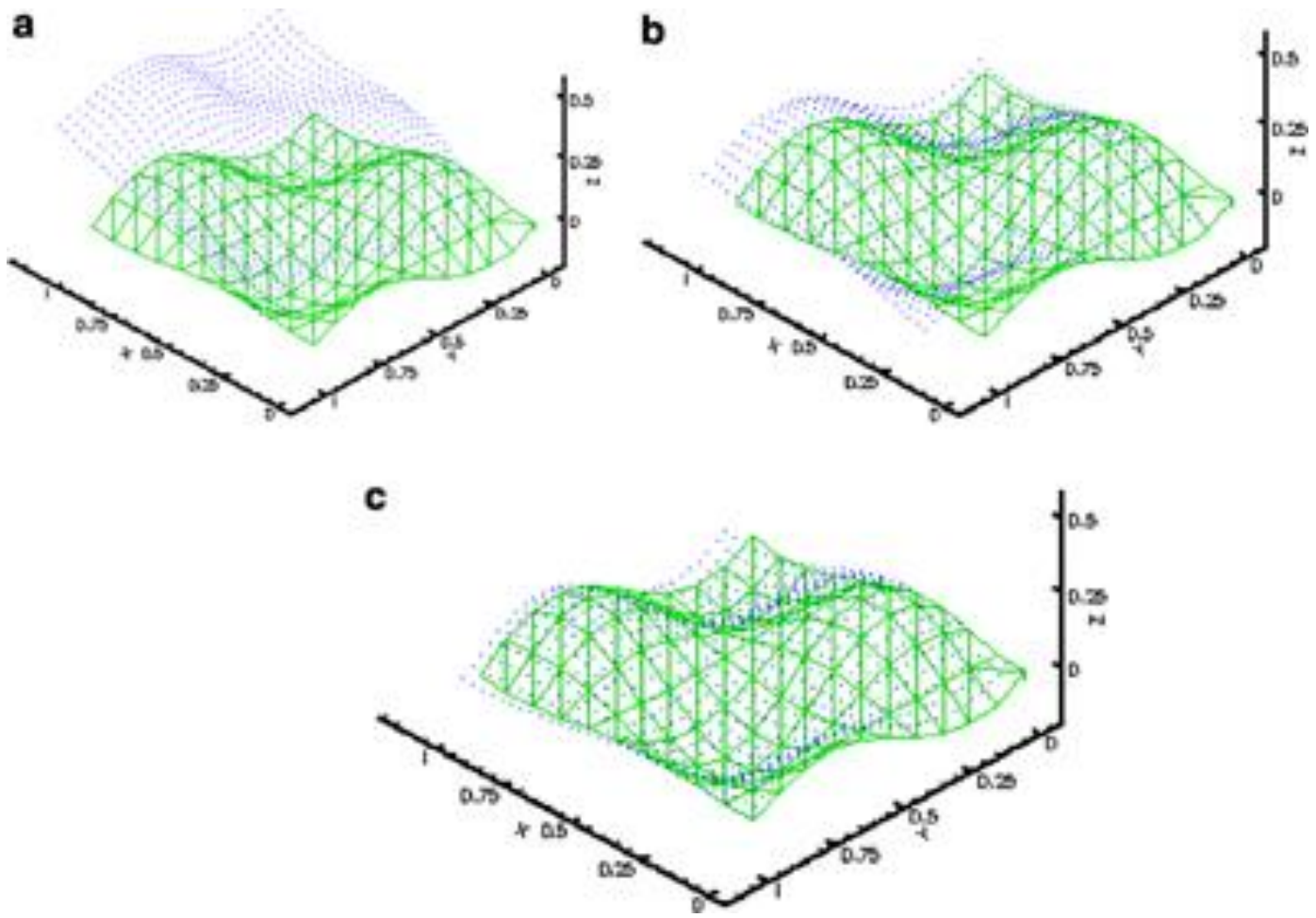

Fig. 8. Alignment between the cloud points and surface to STL format (a) initial position, (b) position after 5 iterations, (c) position after 10 iterations. 

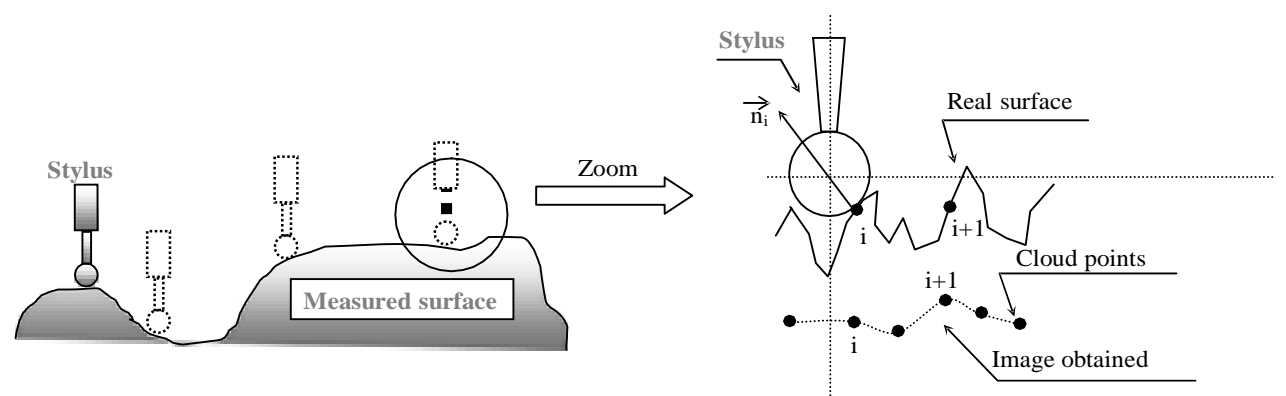

Fig. 11. Measured surface by CMMs

normal vector $\left(\mathrm{n}_{\mathrm{i}}\right)$ and the stylus radius, which generates uncertainty on the real position of the measured point. This uncertainty is even propagated on the inspected surface parameters. Within the same framework, the impact forces attributable to probing are much higher than the generally accepted measurement forces, which can result damage in worked piece (van Vliet \& Schellekens, 1998).

For that, we use a measuring machine equipped with a coaxial optical sensor (CHR 150) for the acquisition phase, which gives the real surface topography.

By this numerical application the tooth which equips the tractor gear box, manufactured in the Engines and Tractors Complex in our country is treated. The gears inspection method used in this company is based on the conventional quality control. Also, the comparison between the conventional inspection gear and the procedure suggested can be established (Fig. 12).

The inspection process of a tooth begins with the acquisition step. To this end, we use a measuring machine equipped with a coaxial optical sensor (CHR 150), whose principle is based on a spectral coding field (Les principes optiques).

This principle of measurement confers two essential qualities:

- Gives an excellent resolution with no influence of ambient lighting.

- The chromatic information coding makes measurement insensitive to the object reflectivity variations and makes it possible to work with the same precision on all materials, transparent, or opaque, polished surface or not.

The acquisition stage was carried out in EA(MS) ${ }^{2}$ Laboratory of the IUT of Aix-en-Provence (France). We recover the points in data files presented as follows (Fig. 13).

At this stage we carry out a graphic rebuilding of the tooth for validation of the acquisition step (Fig. 14).

\section{Conventional}

- Two dimensional

- Standardized section lines of gears flank

- Perpendicular to axis (involute)

- Parallel to axis (lead)

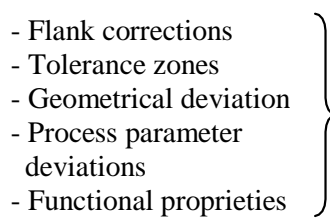

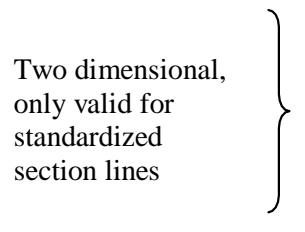

New

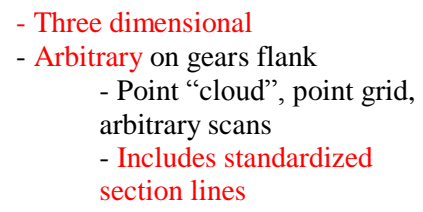

Three dimensional, valid for whole flank

$\Rightarrow$ Improve analysis of manufacturing process $\Rightarrow$ Improve prediction of functionality

Fig. 12. Comparison between conventional and new approach (Goch et al., 2003). 


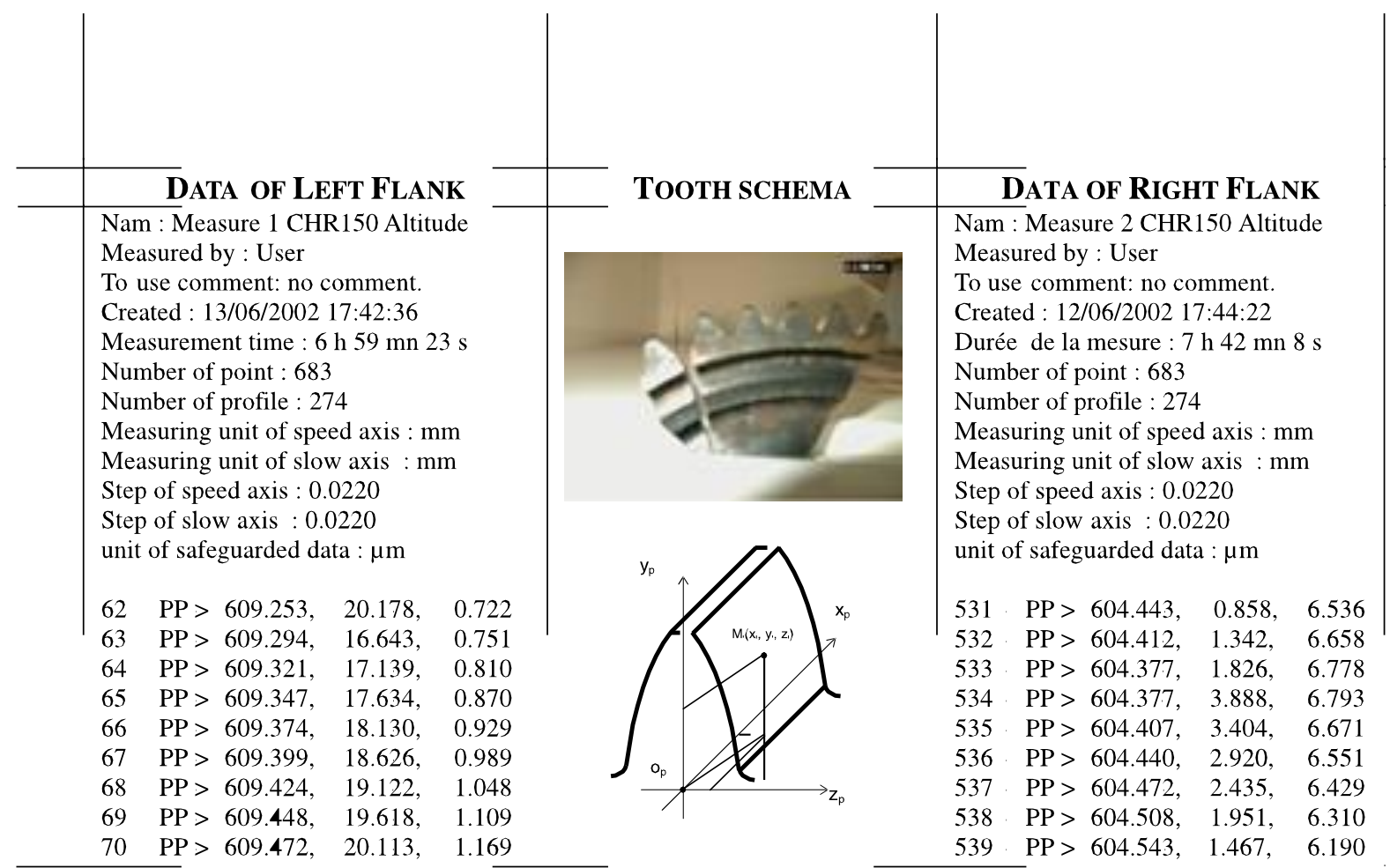

Fig. 13. Parts from the tow data point files.
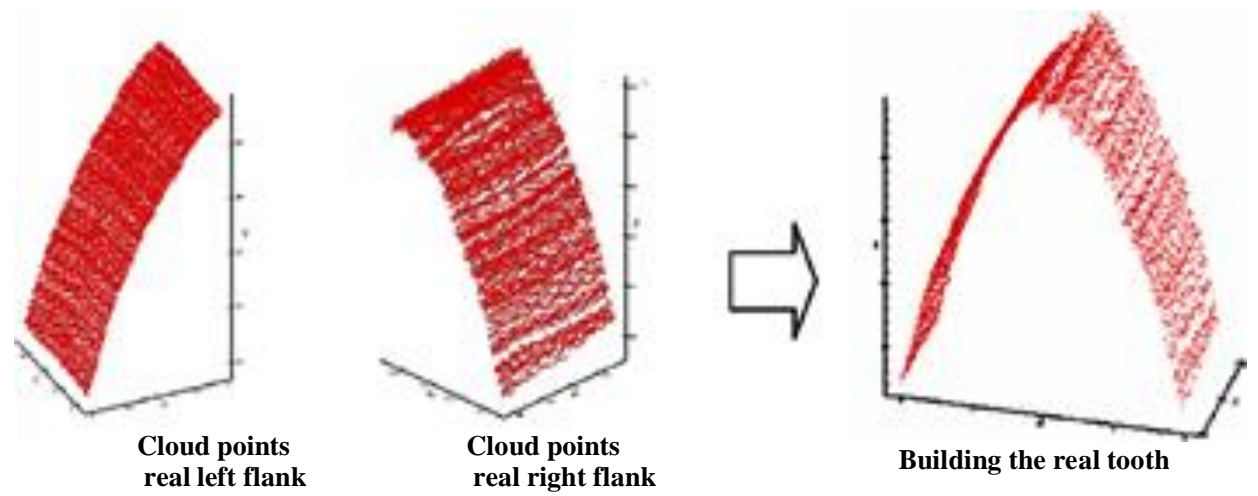

Fig. 14. Rebuilding of the real surface of the tooth.

The ideal model is defined by a generated grid starting from mathematical formulas used for calculating the thickness of the tooth (Sammartini \& Chiff re, 2000). We recover in this case the co-ordinates and the normal of the theoretical points belonging to the involute of circle. We make a triangulation using these points to obtain the ideal model with an STL format (Fig. 15).

The two models being defined we can now call upon the alignment programme of the theoretical set of cloud points with the triangulated surface. The obtained results are presented in Fig. 16.

The errors on the flanks of the tooth are obtained after the alignment process between the real and ideal models is done. This step enables us to obtain the geometric errors cumulated during the manufacturing process, for correction (Fig. 17).

We notice that the diff erence between the real model and the ideal model varies from $-0,085$ to 0,088 [mm] while passing by a median value equal to $0,002[\mathrm{~mm}]$. The interval including negative values corresponds to hollows; on the other hand the interval corresponding to positive values represents the peaks (Fig. 18).

These errors are mainly caused by a grinding device drift during the active surfaces correction phase of the studied tooth (Tsai, Lin, \& Tsai, 1998). In these various zones the contact area will be reduced inducing an increase in the contact pressure at the peaks, generating an accelerated deterioration by crushing the tooth flanks (Abersek, Flasker, \& Glode, 2004). For that, the tooth flank modifications are important structural 


$$
\begin{gathered}
E_{i}=r_{i}\left[\begin{array}{l}
E \\
r
\end{array}+2 .\left(i n v \alpha \square i n v \alpha_{i}\right)\right] \\
\text { With }: i n v \alpha=\tan ( \\
\alpha) \square \alpha \\
-{ }_{2} E=\pi \cdot M
\end{gathered}
$$

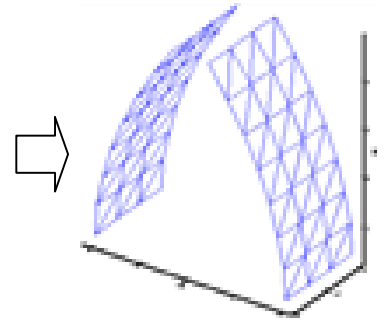

Fig. 15. Theoretical model of the tooth.
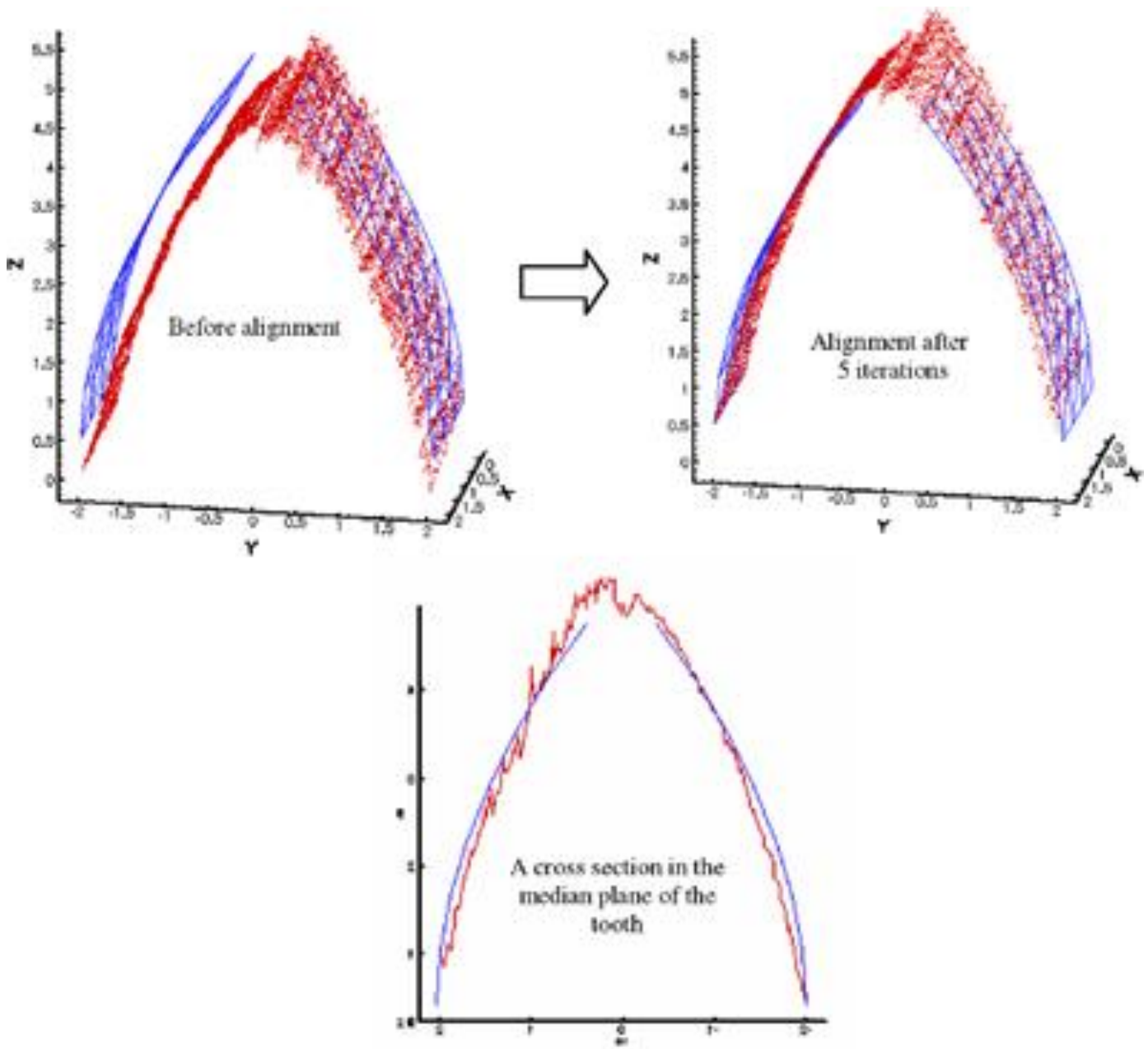

Fig. 16. Alignment between ideal model and the cloud points.

elements of gear manufacturing. The reduce noise and improve running properties as well as enhance loadcarry capability of modern power gears (Henlich \& Linke, 2002). In this manner, the displacement sensitivity, the impact of engagement and the intensifications of contact stress can be reduced. However, the evaluation of the gear quality is still based on unmodified gear flank topography (Goch et al., 2003). Hence, the specifications of corresponding zones of tolerance for the individual quality levels do not consider tooth flank modifications and their eff ects on the running properties of the gear sets. This leads to an insuffi cient function-oriented and manufacturing-oriented design (Goch et al., 2002). Therefore, no suitable metrological 


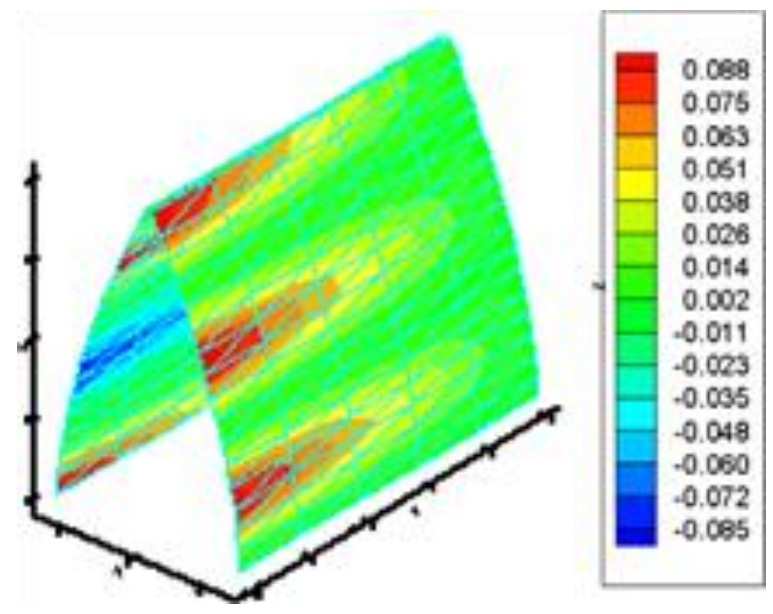

Fig. 17. Errors of form on the flanks of the tooth in $\mathrm{mm}$.

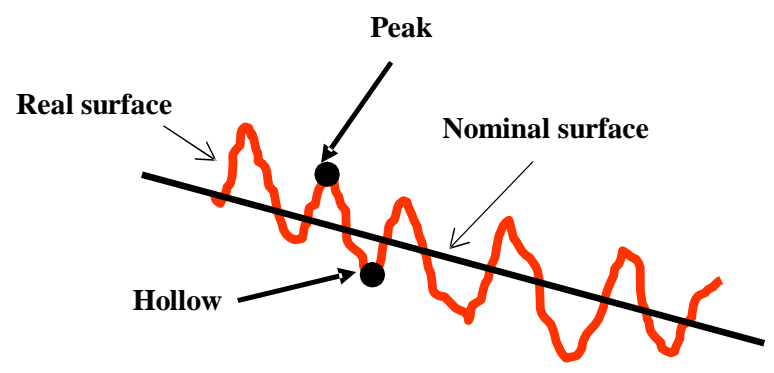

Fig. 18. Topography of activate surface tooth.

parameters for the development of quality control loops in gear manufacturing are available. The consequences are often an insuffi cient process-capability and excessive quality specifications, both resulting in high costs (Pfeifer, Napierala, \& Mandt, 2002). It takes into account that the functional quality of toothing can not be evaluated upon the basis of geometrical measurements alone. Instead, for quality criteria a function-oriented evaluation of the individual toothing properties shall be enabled using the characteristic form deviations of the modified flanks.

\section{Conclusion}

Design and manufacture of complex parts became a current practice in industry; but they are related to requirements at the impossibility of manufacturing with perfect geometries, which is due particularly to the inevitable inaccuracy of the manufacturing processes (Chen, 2002). So the inspection function which consists on the comparison between measurement results and the theoretical parts definition became a veritable defiance; because the final objective of inspecting does not consist in the evaluation of errors but in their elimination or at least to reduce them to tolerable values. For that, it is necessary to act simultaneously on the product and the process in order to obtain an acceptable quality level.

In current state of the metrology software, the inspection of elementary surfaces (plane, cylinder, cone, etc) is not a problem, and most CMMs correct remaining alignment deviations numerically. On the other hand the inspection of surfaces which have geometries of a higher complexity like gears, sculptured surfaces etc. represents a major challenge (Goch \& Tschudi, 1992).

Accordingly to that the undertaken work is placed and consists to establish a procedure for modelling and inspecting complex surfaces with an aim of correction relative deviations in the manufacturing processes. 
The method used is based on the alignment technique by using the ICP algorithm. A numerical investigating considering one tooth of the toothed wheel which equips tractor gearing box is presented.

\section{References}

Abersek, B., Flasker, J., \& Glode, S. (2004). Review of mathematical and experimental models for determination of service life of gears. Engineering Fracture Mechanics, 71, 439-453.

Bergevin, R., Laurendeau, D., \& Poussart, D. (1995). Registering range views of multipart objects. Computer Vision and Image Understanding, 61, 1-16.

Besl, P., \& McKay, N. (1992). A method for registration of 3-D shapes. IEEE Transactions on Pattern Analysis and Machine Intelligence, 14(2), 239-256.

Chen, Mu-chen (2002). Roudness measurements for discontinuous perimeters via machine vision. Computers in Industry, 47, $185-197$.

Chetverikov, Dmitry, Stepanov, Dmitry, \& Krsek, Pavel (2005). Robust Euclidean alignment of 3D point sets: the trimmed iterative closest point algorithm. Image and Vision Computing, 23, 299-309.

Eggert, D., Lorusso, A., \& Fisher, R. (1997). Estimating 3-D rigid body transformations: a comparison of four major algorithms. International Journal of Machine Vision and Applications, 9, 272-290.

Goch, G., \& Günther, A. (2002). Future gear metrology, superficial description and inspection of flanks. International Conference on Gears, 2 (pp. 751-768). VDI-Berichte 1665.

Goch, G. (2003). Gear metrology, keynote papers. Annals of the CIRP, 52(2), 1-37.

Goch, G., \& Tschudi, U. (1992). A universal algorithm for alignment of sculptured surfaces. Annals of the CIRP, Vol. 41/1(1), 597-600.

Henke, R. P., Summerhays, K. D., Baldwin, J. M., Cassou, R. M., \& Brown, C. W. (1999). Methods for evaluation of systematic geometric deviations in machined parts and their relationships to process variables. Precision Engineering, 23, $273-292$.

Henlich, Th., \& Linke, H. (2002). Innovative method for optimising flank modification on toothings. International Conference on Gears, 1 , 167-176, VDI-Berichte 1665.

Kaneko, Shun'ichi, Kondo, Tomonori, \& Miyamoto, Atsushi (2003). Robust matching of 3D contours using iterative closest point algorithm improved by M-estimation. Journal of the Pattern Recognition Society, 36, 2041-2047.

Lai, Jiing-Yih, \& Ueng, Wen-Der (2000). Reconstruction of surfaces of revolution from measured points. Computers in Industry, 41, $147-161$.

Les principes optiques, société STIL S.A., Aix-en-provence, France. Available from www.stilsa.com.

Ma, B., \& Ellis, R. E. (2003). Robust registration for computer-integrated orthopedic surgery: laboratory validation and clinical experience. Medical Image Analysis, 7, 237-250.

Moron, V. (1996). Mise en correspondance de données 3D avec un modèle C.A.O : application à l'inspection automatique. Thèse de doctorat. INSA de Lyon, décembre.

N. N. (1980). "Universal software UMESS und UMESS-S", Firmendrduck der Fa. Carl-Zeiss, Oberkochen.

Pfeifer, T., Napierala, A., \& Mandt, D. (2002). function-oriented evaluation of modified tooth flanks. International Conference on Gears, 2 (769-784). VDI-Berichte 1665.

Pommer, A. (2002). Gear ROLLSCAN for high speed gear measurement. A Revolutionary new method for a complete topographical inspection of gears. International conference on gears, 2 (785-796). VDI-Berichte 1665.

Portman, V. T., \& Shuster, V. G. (1997). Layout errors of machine tools. International Journal Machine Tools Manufacturing, 37(10), 1485-1497.

Rousseuw, P. J., \& Leroy, A. M. (1987). Robust regression and outlier detection. New York: John Wiley \& Sons, Wiley Series in Probability and statistics, ISBN: 0471852333.

Rusinkiewicz, S. \& Levoy, M. (2001). Effi cient variants of the ICP algorithm. In: Proceeding of the 3rd IEEE International Conference on 3-D Digital Imaging and Modeling (145-152). Quebec.

Sablatnig, Robert, \& Kampel, Martin (2002). Model-based registration of front- and backviews of rotationally symmetric objects. Computer Vision and Image Understanding, 87, 90-103.

Sammartini, Maria Pia, \& Chiff re, Leonardo De (2000). Development and validation of a new reference cylindrical gear for pitch measurement. Precision Engineering, 24, 302-309.

Tholath, Jackson, \& Radhakrishnan, V. (1999). Three-dimensional filtering of engineering surfaces using envelope system. Precision Engineering, 23, 221-228.

Tsai, Ming-Haung, Lin, Sheng-Feng, \& Tsai, Ying-Chien (1998). Diagnoses of the operational errors of a threaded-wheel grinder in the grinding of spur gears using kinematic transmission errors. Journal of Materials Processing Technology, 75, 190-197.

Tucker, T. M., \& Kurfess, T. R. (2003). Newton method for parametric surface registration, Part I. Theory (35). Elsevier, computer-aided design.

van Vliet, Wim P., \& Schellekens, Peter H. J. (1998). Development of a fast mechanical probe for coordinate measuring machines. Precision Engineering, 22(03), 141-152. 